\title{
A continuum thermal stress theory for crystals based on interatomic potentials
}

\author{
LIU XiaoLei, TANG QiHeng ${ }^{*}$ \& WANG TzuChiang ${ }^{*}$ \\ State Key Laboratory of Nonlinear Mechanics, Institute of Mechanics, Chinese Academy of Sciences, Beijing 100190, China
}

Received July 12, 2013; accepted September 27, 2013; published online December 30, 2013

\begin{abstract}
This paper presents a new continuum thermal stress theory for crystals based on interatomic potentials. The effect of finite temperature is taken into account via a harmonic model. An EAM potential for copper is adopted in this paper and verified by computing the effect of the temperature on the specific heat, coefficient of thermal expansion and lattice constant. Then we calculate the elastic constants of copper at finite temperature. The calculation results are in good agreement with experimental data. The thermal stress theory is applied to an anisotropic crystal graphite, in which the Brenner potential is employed. Temperature dependence of the thermodynamic properties, lattice constants and thermal strains for graphite is calculated. The calculation results are also in good agreement with experimental data.
\end{abstract}

thermal stress theory, interatomic potential, thermal strain, finite temperature

PACS number(s): 81.05.Bx, 65.40.-b, 47.11.Mn

Citation: Liu X L, Tang Q H, Wang T C. A continuum thermal stress theory for crystals based on interatomic potentials. Sci China-Phys Mech Astron, 2014, 57: 208-217, doi: 10.1007/s11433-013-5371-3

\section{Introduction}

A wide range of applications of nanostructure and nanomaterial have been reported in refs. [1-4]. The characteristic length of the systems of nanomaterial and nanostructure is about several tens of nanometers, while nanoscale effects, such as quantum effects, material defects and surface effects become significant. As a result, classical continuum theory may not be directly applicable for nanosystems. Although the characteristic length is several tens of nanometers, the entire system could still be of the order of micrometers. In this case, ab-initial calculation, molecular dynamics or Monte Carlo simulation which can provide reasonable atomistic analysis is not suitable because of the expensive computation.

In order to capture the essential features of atomistic

\footnotetext{
*Corresponding authors (TANG QiHeng, email: qhtang@imech.ac.cn; WANG
} TzuChiang, email: tcwang@imech.ac.cn) physics while retaining the efficiency of continuum models, many multiscale methods and atomistic-based continuum theories have been proposed in refs. [5-11].

Tadmor et al. [12,13] and Shenoy et al. [14] developed the quasicontinuum $(\mathrm{QC})$ method which combines continuum finite element method with atomistic physics. However, it is restricted to zero temperature. The QC method was extended to take into account the effect of finite temperature under local quasiharmonic approximation [15-17]. Shenoy et al. [15] presented a derivation of an effective energy function to perform Monte Carlo simulation in a mixed atomistic and continuum setting, namely, QC Monte Carlo (QCMC) method.

Dupuy et al. [17] developed a coarse-grained alternative for molecular dynamics by employing the local quasiharmonic model (LQHM). Tang and Aluru [18] established a multiscale model based on finite element method for mechanical analysis of silicon nanostructures at finite temperature. Within the framework of local quasiharmonic model, 
Jiang et al. [19] studied bulk thermodynamic properties of graphite and diamond using their finite-temperature continuum theory developed in terms of the interatomic potential. Tang et al. [20] and Zhao et al. [21] investigated three quasiharmonic models, namely, QHM (quasiharmonic model), QHMK (quasiharmonic model in reciprocal space) and LQHM.

The purpose of this paper is to establish a continuum thermal stress theory for crystals at finite temperature directly from the interatomic potential with low computational cost and high accuracy. The thermodynamic properties, lattice constants, thermal strains, elastic constants and constitutive relation for copper and graphite are calculated respectively. The results have been found in good agreements with the experimental data.

\section{A continuum thermal stress theory for cubic crystals}

The thermal properties of crystal materials may be analyzed by the lattice wave theory with vibration frequency $\omega$. The concept of the thermal strain is introduced and the new thermal stress theory is proposed in this section. The characteristic of new theory is to use the thermal strain instead of frequency of the lattice wave, which made it convenient in practical application $[22,23]$.

\subsection{Vibration frequencies}

The force acting on atom $i$ equals

$$
\begin{aligned}
& \boldsymbol{f}_{i}=-\frac{\partial U_{t o t}}{\partial \boldsymbol{r}_{i}}=-\sum_{j \in \Omega_{i}} \frac{\partial U_{t o t}}{\partial r_{i j}} \frac{\partial r_{i j}}{\partial \boldsymbol{r}_{i}}=\sum_{j \in \Omega_{i}} f_{i j} \boldsymbol{e}_{i j}, \\
& \boldsymbol{e}_{i j}=\frac{\boldsymbol{r}_{i j}}{r_{i j}}, \quad f_{i j}=\frac{\partial U_{t o t}}{\partial r_{i j}},
\end{aligned}
$$

where $\boldsymbol{e}_{i j}$ is the unit vector from atom $i$ to atom $j, r_{i j}$ is the bond length between atom $i$ and atom $j, \Omega_{i}$ represents the set of all atoms which interact with the atom $i, f_{i j}$ is the interaction force between atom $i$ and atom $j$, and $U_{\text {tot }}$ is the total energy stored in atomic bonds of the system.

The dynamic equation of atom $k$ is given by Tang and Wang [22]

$$
\begin{aligned}
& m_{k} \ddot{\boldsymbol{u}}_{k}=\sum_{j \in \Omega_{k}} \boldsymbol{K}_{k j} \cdot\left(\boldsymbol{u}_{j}-\boldsymbol{u}_{k}\right), \\
& \boldsymbol{K}_{k j}=\frac{f_{k j}\left(r_{k j}\right)}{r_{k j}} \boldsymbol{I}+\left(f_{k j}^{\prime}\left(r_{k j}\right)-\frac{f_{k j}\left(r_{k j}\right)}{r_{k j}}\right) \boldsymbol{e}_{k j} \otimes \boldsymbol{e}_{k j},
\end{aligned}
$$

where $\boldsymbol{u}_{j}$ is the displacement of atom $j, \boldsymbol{I}$ is the second-order unit tensor, and $\otimes$ is the tensor multiplication operator.
The solution to eq. (2) can be written as:

$$
\boldsymbol{u}_{j}=\boldsymbol{A}_{j} \mathrm{e}^{\mathrm{i}\left(\omega t-\boldsymbol{r}_{j} \cdot \boldsymbol{q}\right)}
$$

For a crystal with dimensions $N_{1} \boldsymbol{a}_{1}, \quad N_{2} \boldsymbol{a}_{2}$ and $N_{3} \boldsymbol{a}_{3}$ along the three axes, $\boldsymbol{a}_{1}, \boldsymbol{a}_{2}$ and $\boldsymbol{a}_{3}$ are the three basis vectors of Bravais lattice. The Born-Karman boundary condition [24] is applied.

Substituting eq. (3) into eq. (2), one obtains

$$
-m_{k} \boldsymbol{A}_{k} \omega^{2}=\sum_{j \in \Omega_{k}} \boldsymbol{K}_{k j} \cdot\left(\boldsymbol{A}_{j} \mathrm{e}^{-\mathrm{i} \boldsymbol{r}_{k j} \cdot \boldsymbol{q}}-\boldsymbol{A}_{k}\right), k=1, \cdots, N,
$$

where $N$ is the total number of atoms in the system.

For copper, per unit cell contains only one atom. All atoms have the same magnitude, $\boldsymbol{A}_{k}=\boldsymbol{A}_{j}=\boldsymbol{A}$.

As a result, each copper atom interacts with 54 neighbors (including the nearest neighbors, second nearest neighbors up to fourth nearest neighbors).

Hence for atom $k$ the characteristic equation can be written as:

$$
\begin{gathered}
\left|m \omega^{2} \boldsymbol{I}+\sum_{j=1}^{54} \boldsymbol{K}_{k j} \cdot\left(\mathrm{e}^{-\mathrm{i} \boldsymbol{r}_{k j} \cdot \boldsymbol{q}}-1\right)\right|=0, \\
\boldsymbol{K}_{k j}=\alpha_{k j} \boldsymbol{I}+\beta_{k j} \boldsymbol{e}_{k j} \otimes \boldsymbol{e}_{k j}, \\
\alpha_{k j}=\frac{f\left(r_{k j}\right)}{r_{k j}}, \quad \beta_{k j}=f^{\prime}\left(r_{k j}\right)-\alpha_{k j} .
\end{gathered}
$$

\subsection{Equilibrium at finite temperature}

The equilibrium bond length is a function of temperature $T$, i.e.

$$
r=r^{(0)}(T),
$$

where the superscript 0 denotes the state only subject to free thermal expansion. That is to say, the pressure acting on the volume element of the crystal equals zero, $p=0$. The quasi equilibrium distance $r^{(0)}(T)$ is determined by minimizing the Helmholtz free energy $A$, i.e.

$$
\begin{aligned}
& p=-\frac{\partial A}{\partial V}=0, \\
& A=U_{t a t}+T k_{\mathrm{B}} \sum_{j} \ln \left[2 \sinh \left(\hbar \omega_{j} / 2 k_{\mathrm{B}} T\right)\right],
\end{aligned}
$$

where $V$ is the volume, $k_{\mathrm{B}}$ is the Boltzmann constant, $\hbar$ is the Planck's constant and $\omega_{j}$ is the vibration frequency of the atom system.

\subsection{Stress-strain relationship}

The thermal stress theory for crystals subject to arbitrary 
deformation is presented in this section. The homogeneous deformation measures can be related to the motion of many atoms via the Cauchy-Born rule, which equates the strain energy to the energy stored in atomic bonds and the vibration energy.

Figures 1(a)-(c) are the initial configuration, intermediate configuration and current configuration, respectively.

The initial configuration is the state of undeformed solid at absolute zero temperature. The deformation gradient $\boldsymbol{F}^{*}$ is the free thermal expansion as temperature rises to $T$. As a result, the intermediate configuration is in the zero-stress state.

Since $F^{e}$ is the elastic deformation gradient from the intermediate configuration to the current configuration, the second Piola-Kirchhoff stress can be determined by

$$
\boldsymbol{S}=\frac{\partial W}{\partial \boldsymbol{E}^{e}}=\frac{1}{V^{*}} \frac{\partial U_{t o t}\left(\boldsymbol{E}^{e}\right)}{\partial \boldsymbol{E}^{e}},
$$

where $W$ is the strain energy density, $\boldsymbol{E}^{e}$ is the elastic Green strain tensor, and $V^{*}$ is the volume at the intermediate configuration.

The Cauchy stress takes the form:

$$
\boldsymbol{\sigma}=\frac{1}{V} \boldsymbol{F}^{e}\left\{\frac{\partial U\left(\boldsymbol{E}^{e}\right)}{\partial \boldsymbol{E}^{e}}\right\} \boldsymbol{F}^{\mathrm{eT}},
$$

where $V$ is the volume at the current configuration.

The total deformation gradient is

$$
\boldsymbol{F}=\boldsymbol{F}^{e} \boldsymbol{F}^{*} \text {. }
$$

The thermal strain tensor, elastic strain tensor and total

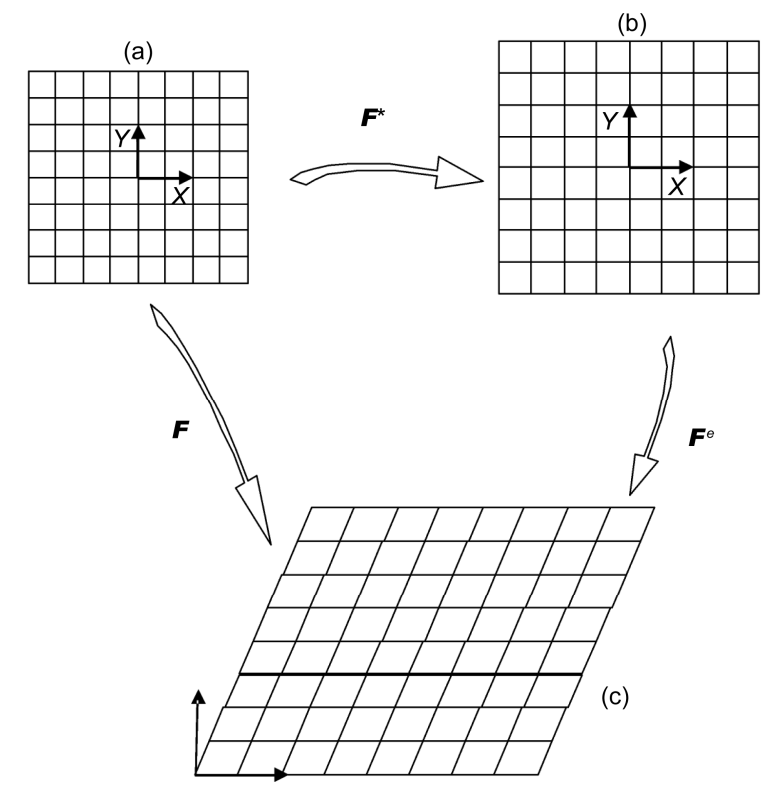

Figure 1 Schematic diagram of deformations. (a) is the initial configuration. (b) is the intermediate configuration. (c) is the current configuration. strain tensor take the forms respectively

$$
\begin{aligned}
\boldsymbol{E}^{*} & =\left(\boldsymbol{F}^{* \mathrm{~T}} \boldsymbol{F}^{*}-\boldsymbol{I}\right) / 2, \\
\boldsymbol{E}^{e} & =\left(\boldsymbol{F}^{e \mathrm{~T}} \boldsymbol{F}^{e}-\boldsymbol{I}\right) / 2, \\
\boldsymbol{E} & =\left(\boldsymbol{F}^{\mathrm{T}} \boldsymbol{F}-\boldsymbol{I}\right) / 2 .
\end{aligned}
$$

Substituting eq. (10) into eq. (11c), one obtains

$$
\begin{aligned}
\boldsymbol{E} & =\frac{1}{2}\left[\left(\boldsymbol{F}^{e} \boldsymbol{F}^{*}\right)^{\mathrm{T}} \boldsymbol{F}^{e} \boldsymbol{F}^{*}-\boldsymbol{I}\right] \\
& =\frac{1}{2}\left[\boldsymbol{F}^{* \mathrm{~T}} \boldsymbol{F}^{e \mathrm{~T}} \boldsymbol{F}^{e} \boldsymbol{F}^{*}-\boldsymbol{I}\right] \\
& =\frac{1}{2}\left[\boldsymbol{F}^{* \mathrm{~T}}\left(\boldsymbol{I}+2 \boldsymbol{E}^{e}\right) \boldsymbol{F}^{*}-\boldsymbol{I}\right] \\
& =\frac{1}{2}\left(\boldsymbol{F}^{* \mathrm{~T}} \boldsymbol{F}^{*}-\boldsymbol{I}\right)+\boldsymbol{F}^{* \mathrm{~T}} \boldsymbol{E}^{e} \boldsymbol{F}^{*} \\
& =\boldsymbol{E}^{*}+\boldsymbol{F}^{* \mathrm{~T}} \boldsymbol{E}^{e} \boldsymbol{F}^{*} .
\end{aligned}
$$

From eq. (12), one obtains

$$
\boldsymbol{F}^{* \mathrm{~T}} \boldsymbol{E}^{e} \boldsymbol{F}^{*}=\boldsymbol{E}-\boldsymbol{E}^{*} .
$$

Let us consider the Polar decomposition of the tensor. The deformation gradients $\boldsymbol{F}, \boldsymbol{F}^{*}$ and $\boldsymbol{F}^{e}$ can be rewritten respectively as:

$$
\begin{aligned}
& \boldsymbol{F}=\boldsymbol{R} \boldsymbol{U}, \\
& \boldsymbol{F}^{*}=\boldsymbol{R}^{*} \boldsymbol{U}^{*}, \\
& \boldsymbol{F}^{e}=\boldsymbol{R}^{e} \boldsymbol{U}^{e},
\end{aligned}
$$

where $\boldsymbol{R}, \quad \boldsymbol{R}^{*}$ and $\boldsymbol{R}^{e}$ are the rotation tensors; $\boldsymbol{U}, \boldsymbol{U}^{*}$ and $\boldsymbol{U}^{e}$ are the stretch tensors. Without loosing generality, one can assume $R^{*}=\boldsymbol{I}$. Then from eq. (13), one can obtain

$$
\boldsymbol{E}^{e}=\left(\boldsymbol{U}^{* \mathrm{~T}}\right)^{-1}\left(\boldsymbol{E}-\boldsymbol{E}^{*}\right)\left(\boldsymbol{U}^{*}\right)^{-1} .
$$

Therefore, Cauchy stress in eq. (9) now becomes

$$
\begin{aligned}
& \boldsymbol{\sigma}=\frac{1}{V} \boldsymbol{F}^{e}\left\{\left[\frac{\partial U\left(\boldsymbol{E}^{e}\right)}{\partial \boldsymbol{E}^{e}}\right]\right\} \boldsymbol{F}^{e \mathrm{~T}}, \\
& \boldsymbol{E}^{e}=\left(\boldsymbol{U}^{* \mathrm{~T}}\right)^{-1}\left(\boldsymbol{E}-\boldsymbol{E}^{*}\right)\left(\boldsymbol{U}^{*}\right)^{-1},
\end{aligned}
$$

If the thermal strain $\boldsymbol{E}^{*}$ is small, one can get the following equation:

$$
\boldsymbol{\sigma}=\frac{1}{V} \boldsymbol{F}^{e}\left\{\left[\frac{\partial U\left(\boldsymbol{E}^{e}\right)}{\partial \boldsymbol{E}^{e}}\right]_{\boldsymbol{E}^{e}=\boldsymbol{E}-\boldsymbol{E}^{*}}\right\} \boldsymbol{F}^{e \mathrm{~T}} .
$$

Eq. (17) is the new thermal stress theory. There are no temperature $T$ and no thermal vibration frequency $\omega_{j}$ of any atom $j$ appearing in eq. (17), but the thermal strain $\boldsymbol{E}^{*}$ is found in eq. (17) instead. Compared to the previous thermal stress theory [22], eq. (17) is much more convenient and 
effective in practical application.

Eq. (11a) can be rewritten as:

$$
\boldsymbol{E}^{*}=\left[\left(\boldsymbol{U}^{*}\right)^{2}-\boldsymbol{I}\right] / 2 .
$$

Then, we obtain the Taylor expansion of $\boldsymbol{U}^{*}$,

$$
\boldsymbol{U}^{*}=\left(\boldsymbol{I}+2 \boldsymbol{E}^{*}\right)^{\frac{1}{2}}=\boldsymbol{I}+\boldsymbol{E}^{*}-\frac{1}{2}\left(\boldsymbol{E}^{*}\right)^{2}+\cdots
$$

If the thermal strain $\boldsymbol{E}^{*}$ is small, one can get the following equation:

$$
\boldsymbol{U}^{*} \cong \boldsymbol{I}+\boldsymbol{E}^{*}
$$

\section{Calculation results for copper}

\subsection{EAM potential for copper}

In order to obtain valuable results, one should choose an accurate interatomic potential. The EAM potential proposed by Mishin et al. [25] for copper is adopted in this paper.

\subsection{Thermodynamic properties of copper}

The calculations are carried out for $10 \times 10 \times 10,20 \times 20 \times 20$ and $100 \times 100 \times 100$ unit cells with periodic boundary conditions.

\subsubsection{Specific heat and bulk modulus}

The specific heat $C_{V}$ is given in ref. [26]

$$
\begin{aligned}
& E=\sum_{\boldsymbol{q}} \sum_{s=1}^{3}\left(\frac{1}{2} \hbar \omega_{s}(\boldsymbol{q})+\frac{\hbar \omega_{s}(\boldsymbol{q})}{\mathrm{e}^{\hbar \omega_{s}(\boldsymbol{q}) / k_{\mathrm{B}} T}-1}\right) \\
& C_{V}=\frac{\mathrm{d} E(T)}{\mathrm{d} T}=\sum_{q} \sum_{s=1}^{3} k_{\mathrm{B}} \frac{\left(\hbar \omega_{s}(\boldsymbol{q}) / k_{\mathrm{B}} T\right)^{2} \mathrm{e}^{\hbar \omega_{s}(\boldsymbol{q}) / k_{\mathrm{B}} T}}{\left(\mathrm{e}^{\hbar \omega_{s}(\boldsymbol{q}) / k_{\mathrm{B}} T}-1\right)^{2}},
\end{aligned}
$$

where $E$ is the total thermal energy; $\omega_{s}(q)$ is the vibration frequency of the crystal lattice.

Figure 2 shows the comparison of the present calculation results with the experimental data in ref. [27]. One can see that the present calculation results are in good agreement with experimental data for temperatures up to $400 \mathrm{~K}$. For higher temperatures, anharmonic effect becomes a little more significant.

$$
\text { According to } K_{0}=V_{0}\left(\frac{\mathrm{d}^{2} U_{t o t}}{\mathrm{~d} V^{2}}\right)_{V_{0}}[28] \text {, the bulk modulus }
$$

of crystal can be calculated, where $V_{0}$ is the volume prior to deformation and $U_{\text {tot }}$ is the total potential energy.

The calculation result is $K_{0}=138 \mathrm{GPa}$, which is in good agreement with experimental data $137 \mathrm{GPa}$ [28].

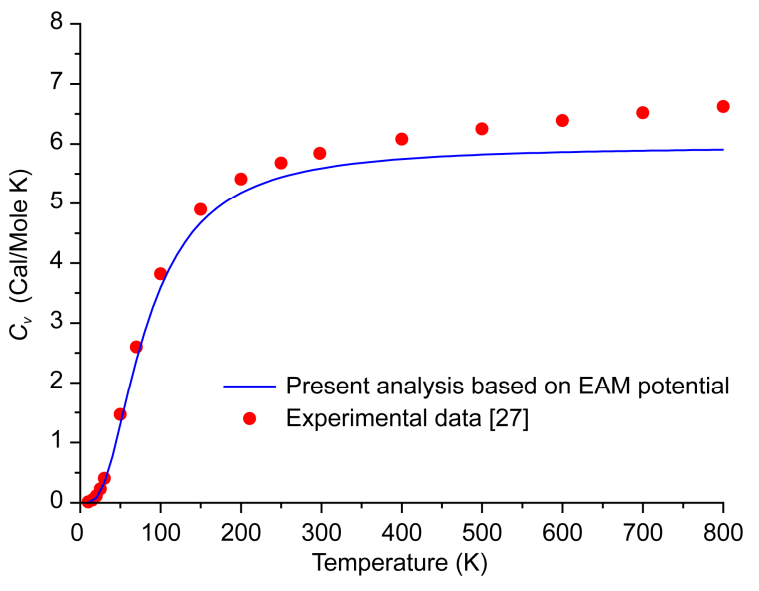

Figure 2 (Color online) Temperature dependence of specific heat $C_{V}$ for copper predicted by eq. (21). The experimental data of copper [27] are also shown.

\subsubsection{CTE of copper}

According to the Grüneisen's law, the coefficient of thermal expansion (CTE) $\alpha$ of copper is given by

$$
\alpha=\frac{1}{3} \alpha_{V}=\frac{\gamma}{3 K_{0}} \frac{C_{V}}{V}
$$

where $\gamma$ is the Grüneisen's parameter, $K_{0}$ is the bulk modulus of the crystal, $V$ is the volume and $C_{V}$ is the specific heat. For copper, $\gamma$ is a constant and taken to be 2.0.

The calculation results for the coefficient of thermal expansion (CTE) $\alpha$ are shown in Figure 3. The present result agrees quite well with the experimental data [28] for temperatures up to $400 \mathrm{~K}$.

\subsection{Thermal strain and lattice constant}

Two methods are used to calculate the thermal strain and

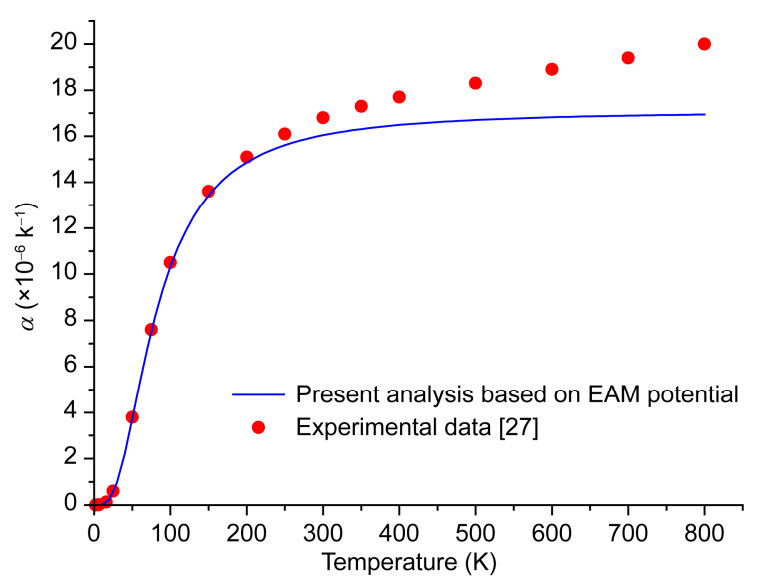

Figure 3 (Color online) Temperature dependence of the coefficient of thermal expansion $\alpha$ for copper predicted by eq. (22). The experimental data of copper [27] are also shown for comparison. 
lattice constant. In method 1 , the thermal strain is given by

$$
\varepsilon_{T}=\int_{T_{0}}^{T} \alpha \mathrm{d} T,
$$

where $T_{0}$ is often chosen to be room temperature, $\varepsilon_{T}$ is the thermal strain from temperature $T_{0}$ to temperature $T$ when the crystal is expanding freely.

The lattice constant after thermal expansion is given by Jiang et al. [19]

$$
r^{(0)}(T)=r^{(0)}\left(T_{0}\right)\left(1+\int_{T_{0}}^{T} \alpha \mathrm{d} T\right),
$$

where $\alpha$ is CTE and $r^{(0)}\left(T_{0}\right)$ is the lattice constant at room temperature.

In method 2, the lattice constant, $r^{(0)}(T)$ is determined by minimizing the Helmholtz free energy $A, \frac{\partial A}{\partial V}=0$. Thermal strain can be expressed by

$$
\varepsilon_{T}=\frac{r^{(0)}(T)}{r^{(0)}(293 \mathrm{~K})}-1,
$$

where $r^{(0)}(293 \mathrm{~K})$ is the quasi equilibrium distance at room temperature.

The comparison of the present calculation result and experimental data for thermal strain is shown in Figures 4 and 5 . The calculated results of both methods are in good with the experimental data [29].

\subsection{Elastic constants of copper}

The thermal strain tensor $\boldsymbol{E}^{*}(T)$ for copper is given by

$$
\boldsymbol{E}^{*}(T)=\left[\begin{array}{ccc}
\varepsilon_{T}^{(*)} & 0 & 0 \\
0 & \varepsilon_{T}^{(*)} & 0 \\
0 & 0 & \varepsilon_{T}^{(*)}
\end{array}\right],
$$

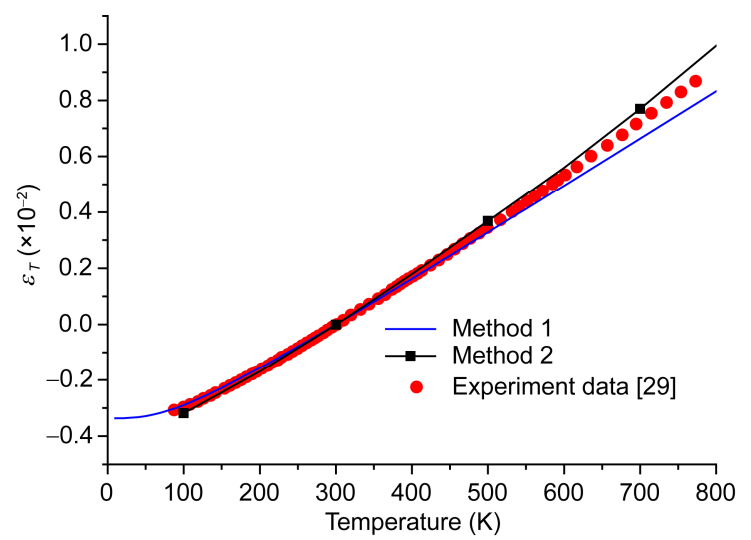

Figure 4 (Color online) Variation of thermal strain with temperature obtained through method 1 and method 2, and the experimental data [29] are also shown for comparison.

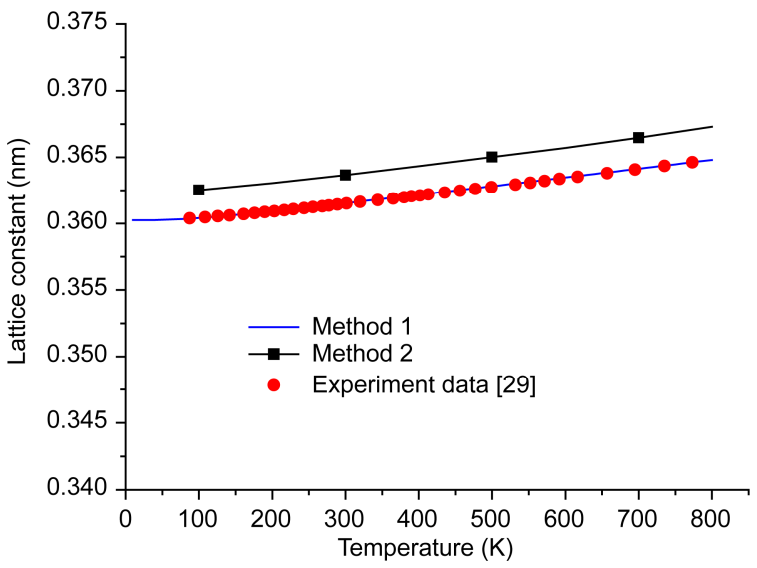

Figure 5 (Color online) Variation of lattice constants with temperature. Calculation results predicted by method 1 and method 2 and the experimental data [29] are shown for comparison.

where $\varepsilon_{T}^{*}$ is the normal thermal strain when the crystal expands freely from absolute zero temperature to $T$,

$$
\varepsilon_{T}^{*}(T)=\int_{0}^{T} \alpha \mathrm{d} T=\int_{0}^{T_{0}} \alpha \mathrm{d} T+\varepsilon_{T}(T) .
$$

The stress-strain relationship of the anisotropic materials in the linear elastic range is defined by

$$
\begin{aligned}
& \sigma_{x}=C_{11} \varepsilon_{x}+C_{12} \varepsilon_{y}+C_{13} \varepsilon_{z}+C_{14} \gamma_{x y}+C_{15} \gamma_{y z}+C_{16} \gamma_{z x}, \\
& \sigma_{y}=C_{21} \varepsilon_{x}+C_{22} \varepsilon_{y}+C_{23} \varepsilon_{z}+C_{24} \gamma_{x y}+C_{25} \gamma_{y z}+C_{26} \gamma_{z x}, \\
& \sigma_{z}=C_{31} \varepsilon_{x}+C_{32} \varepsilon_{y}+C_{33} \varepsilon_{z}+C_{34} \gamma_{x y}+C_{35} \gamma_{y z}+C_{36} \gamma_{z x}, \\
& \tau_{x y}=C_{41} \varepsilon_{x}+C_{42} \varepsilon_{y}+C_{43} \varepsilon_{z}+C_{44} \gamma_{x y}+C_{45} \gamma_{y z}+C_{46} \gamma_{z x}, \\
& \tau_{y z}=C_{51} \varepsilon_{x}+C_{52} \varepsilon_{y}+C_{53} \varepsilon_{z}+C_{54} \gamma_{x y}+C_{55} \gamma_{y z}+C_{56} \gamma_{z x}, \\
& \tau_{z x}=C_{61} \varepsilon_{x}+C_{62} \varepsilon_{y}+C_{63} \varepsilon_{z}+C_{64} \gamma_{x y}+C_{65} \gamma_{y z}+C_{66} \gamma_{z x},
\end{aligned}
$$

where $\sigma_{x}, \sigma_{y}, \sigma_{z}, \tau_{x y}, \tau_{y z}, \tau_{z x}$ are the stress components, $\varepsilon_{x}, \varepsilon_{y}, \varepsilon_{z}, \gamma_{x y}, \gamma_{y z}, \gamma_{z x}$ are the strain components, and other 21 parameters are the elastic constants which depend on temperature $T$.

For single-crystal copper, we have

$$
\begin{aligned}
& C_{11}(T)=C_{22}(T)=C_{33}(T), \\
& C_{12}(T)=C_{13}(T)=C_{23}(T)=C_{21}(T)=C_{31}(T)=C_{32}(T),(28) \\
& C_{44}(T)=C_{55}(T)=C_{66}(T)
\end{aligned}
$$

and the other parameters equal 0 .

There are only three independent elastic parameters, namely $C_{11}(T), C_{12}(T)$ and $C_{44}(T)$. Then, two cases have been investigated to capture three elastic parameters.

In the first case, from the deformation shown in Figure 6(a), suppose a volume element of copper crystal is subject to an elastic uniaxial strain $\varepsilon_{x}=10^{-6}$ from the intermediate configuration to the current configuration as follow: 

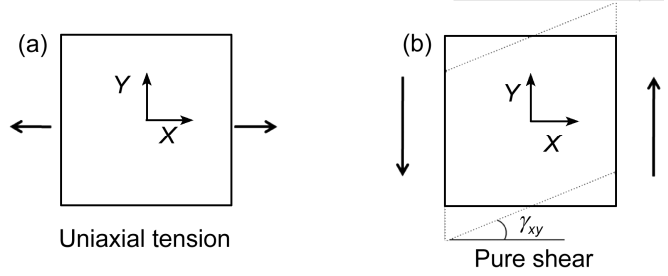

Figure 6 (a) Elastic stretch deformation $\boldsymbol{E}_{\text {strech }}^{\text {e }}$ from the intermediate configuration to the current configuration; (b) elastic shear deformation $\boldsymbol{E}_{\text {shear }}^{\mathrm{e}}$ from the intermediate configuration to the current configuration.

$$
\boldsymbol{E}_{\text {stratch }}^{\mathrm{e}}=\left[\begin{array}{ccc}
10^{-6} & 0 & 0 \\
0 & 0 & 0 \\
0 & 0 & 0
\end{array}\right]
$$

where $\boldsymbol{E}_{\text {stretch }}^{\mathrm{e}}$ is the corresponding elastic strain tensor.

The total strain tensor $\boldsymbol{E}$ from the initial configuration to the current configuration is expressed as:

$$
\boldsymbol{E}=\boldsymbol{E}^{*}+\left(\boldsymbol{U}^{*}\right)^{\mathrm{T}} \boldsymbol{E}^{e} \boldsymbol{U}^{*}
$$

Substituting eqs. (29) and (26) into eq. (16), we have

$$
\sigma_{1}=\left[\begin{array}{ccc}
\sigma_{x}(T) & 0 & 0 \\
0 & \sigma_{y}(T) & 0 \\
0 & 0 & \sigma_{z}(T)
\end{array}\right],
$$

where $\sigma_{1}$ is the corresponding stress tensor.

The elastic constants can be expressed by

$$
\begin{aligned}
& C_{11}(T)=\frac{\sigma_{x}(T)}{\varepsilon_{x}}=10^{6} \times \sigma_{x}(T), \\
& C_{21}(T)=\frac{\sigma_{y}(T)}{\varepsilon_{x}}=10^{6} \times \sigma_{y}(T), \\
& C_{31}(T)=\frac{\sigma_{z}(T)}{\varepsilon_{x}}=10^{6} \times \sigma_{z}(T) .
\end{aligned}
$$

In the second case, from the deformation shown in Figure 6(b), suppose a volume element of copper crystal is subject to an elastic shear strain $\gamma_{x y}=2 \varepsilon_{x y}=10^{-6}$, i.e.

$$
\boldsymbol{E}_{\text {shear }}^{\mathrm{e}}=\left[\begin{array}{ccc}
0 & 0.5 \times 10^{-6} & 0 \\
0.5 \times 10^{-6} & 0 & 0 \\
0 & 0 & 0
\end{array}\right] \text {. }
$$

Substituting eqs. (32) and (26) into eq. (16), we obtain

$$
\boldsymbol{\sigma}_{2}=\left[\begin{array}{ccc}
0 & \tau_{x y}(T) & 0 \\
\tau_{y x}(T) & 0 & 0 \\
0 & 0 & 0
\end{array}\right]
$$

where $\sigma_{2}$ is the corresponding stress tensor.
The $C_{44}$ is obtained as:

$$
C_{44}(T)=\frac{\tau_{x y}(T)}{\gamma_{x y}}=10^{6} \times \tau_{x y}(T) .
$$

In Figure 7, our computational results indicate that the elastic constants of copper decrease with the increase of temperature. For comparison, some experimental results [30] are also displayed. From the good agreement we can once again expect that the prediction for the thermal strain $\varepsilon_{T}$ in sect. 4.4 is quite accurate.

\section{Application of the thermal stress theory to graphite}

\subsection{Vibration frequencies of graphite}

For graphite, the interaction of layer-layer is van der waals force which is quite small compared to the in-plane interaction. As a result, the interaction of layer-layer is ignored in the dynamic equation of the carbon atom. Therefore graphene can be a substitute for graphite when the vibration frequencies of the system are calculated.

As is shown in Figure 8, that carbon atoms in graphene can be classified into two types A and B.

For a two dimensional graphene crystal, wave vector $\boldsymbol{q}$ can be expressed as:

$$
\boldsymbol{q}=\frac{k_{1}}{N_{1}} \boldsymbol{b}_{1}+\frac{k_{2}}{N_{2}} \boldsymbol{b}_{2}, k_{1}=1, \cdots, N_{1}, k_{2}=1, \cdots, N_{2},
$$

where $\boldsymbol{b}_{1}$ and $\boldsymbol{b}_{2}$ are the basis vector of the reciprocal lattice.

With the parameters $R^{(1)}=0.17 \mathrm{~nm}, R^{(2)}=0.2 \mathrm{~nm}$ and the bond length at zero temperature equal to $0.142 \mathrm{~nm}$ respectively, the cutoff function of carbon's Brenner potential $[31,32]$ is

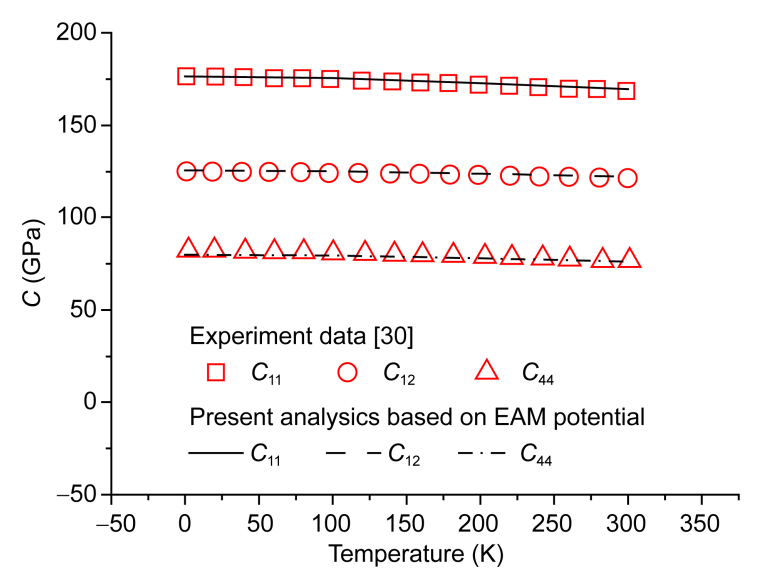

Figure 7 (Color online) Comparison of elastic constants $C_{11}, C_{12}$ and $C_{44}$ between the current analysis and the experimental data [30]. 


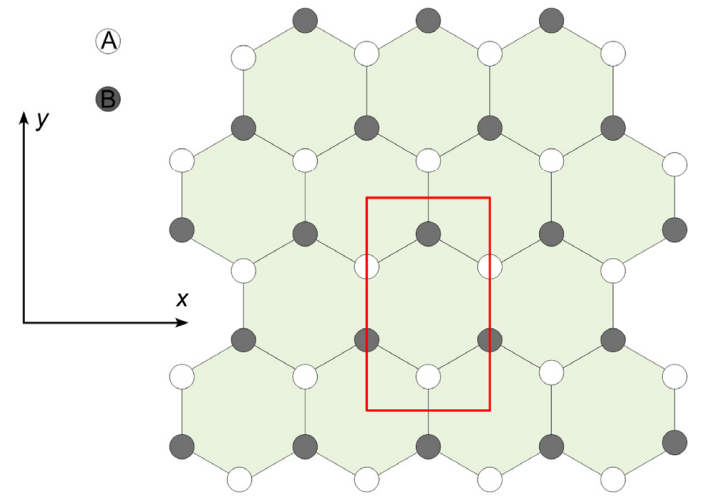

Figure 8 (Color online) A schematic diagram of the atomic structure of a graphene with a representative atom 0 , its three nearest-neighbor atoms 1 , 2 , and 3, and six second-nearest-neighbor atoms 4, 5, 6, 7, 8 and 9. The open circles represent the carbon atom $\mathrm{A}$, and the shaded circles represent the carbon atom $\mathrm{B}$.

$$
\begin{aligned}
& f_{C}\left(r_{i j}\right)= \\
& \begin{cases}1, & r<R^{(1)}, \\
\frac{1}{2}\left\{1+\cos \left[\pi\left(r-R^{(1)}\right) /\left(R^{(2)}-R^{(1)}\right)\right]\right\} & , R^{(1)}<r<R^{(2)}, \\
0, & r>R^{(2)} .\end{cases}
\end{aligned}
$$

As a result, for one carbon atom $\mathrm{A}$, it interacts with 3 nearest neighbours $\mathrm{B}$. The dynamic equations therefore can be expressed by

$$
\begin{aligned}
& m \ddot{\boldsymbol{u}}_{A_{i}}=\sum_{j=1}^{3} K\left(r_{A_{i} B_{j}}\right) \cdot\left(u_{B_{j}}-u_{A_{i}}\right), \\
& m \ddot{\boldsymbol{u}}_{B_{i}}=\sum_{j=1}^{3} K\left(r_{B_{i} A_{j}}\right) \cdot\left(u_{A_{j}}-u_{B_{i}}\right),
\end{aligned}
$$

where $\mathrm{A}$ and $\mathrm{B}$ represent the two types of the atom, and $i$ and $\alpha$ are serial numbers of the atoms.

In graphene, A and B atoms have different amplitudes. The solutions of lattice wave are

$$
\begin{aligned}
& \boldsymbol{u}_{A_{i}}=\boldsymbol{A} \mathrm{e}^{\mathrm{i}\left(\omega t-\boldsymbol{r}_{A_{i}} \bullet \boldsymbol{q}\right)}, \\
& \boldsymbol{u}_{B_{i}}=\boldsymbol{B} \mathrm{e}^{\mathrm{i}\left(\omega t-\boldsymbol{r}_{B_{i}} \bullet q\right)} .
\end{aligned}
$$

Substituting eq. (38) into eq. (37), we get

$$
\left(m \omega^{2} \boldsymbol{I}-\boldsymbol{J}\right)\left(A_{x}, A_{y}, A_{z}, B_{x}, B_{y}, B_{z}\right)^{\mathrm{T}}=\mathbf{0}
$$

where $J$ is a $6 \times 6$ matrix, and $A_{x}, A_{y}, A_{z}, B_{x}, B_{y}$ and $B_{z}$ are the components of the amplitudes of the atoms.

The characteristic equation can be written as:

$$
\left|m \omega^{2} \boldsymbol{I}-\boldsymbol{J}\right|=0 .
$$

Hence we can get six vibration frequencies for each $\boldsymbol{q}$.

\subsection{Specific heat and CTE of graphite}

\subsubsection{Specific heat of graphite}

The $C_{V}$ for graphite can be composed of two components [33]:

$$
C_{V}=\frac{2}{3} C_{V, a}+\frac{1}{3} C_{V, c},
$$

where $C_{V, a}$ is the in-plane specific heat at constant volume, $C_{V, c}$ is the out-of-plane specific heat at constant volume. $C_{V, a}$ and $C_{V, c}$ take the forms:

$$
\begin{aligned}
& C_{V, a}=\sum_{q} \sum_{s=1}^{4} k_{\mathrm{B}} \frac{\left(\hbar \omega_{s}^{a}(\boldsymbol{q}) / k_{\mathrm{B}} T\right)^{2} \mathrm{e}^{\hbar \omega_{s}^{a}(\boldsymbol{q}) / k_{\mathrm{B}} T}}{\left(\mathrm{e}^{\hbar \omega_{s}^{a}(\boldsymbol{q}) / k_{\mathrm{B}} T}-1\right)^{2}}, \\
& C_{V, c}=\sum_{\boldsymbol{q}} \sum_{s=5}^{6} k_{\mathrm{B}} \frac{\left(\hbar \omega_{s}^{c}(\boldsymbol{q}) / k_{\mathrm{B}} T\right)^{2} \mathrm{e}^{\hbar \omega_{s}^{c}(\boldsymbol{q}) / k_{\mathrm{B}} T}}{\left(\mathrm{e}^{\hbar \omega_{s}^{c}(\boldsymbol{q}) / k_{\mathrm{B}} T}-1\right)^{2}},
\end{aligned}
$$

where $\omega_{s}^{a}(\boldsymbol{q})$ is the in-plane frequency, $\omega_{s}^{c}(\boldsymbol{q})$ is the out-of-plane frequency.

Figure 9 shows the comparison of specific heat versus temperature for graphite, it is obvious that the predicted specific heat agrees very well with experimental data [27] for graphite.

\subsubsection{CTE of graphite}

Since thermal expansion of graphite is anisotropic, the expression of Grüneisen's law for graphite is different from that for copper. As pointed by Morgan [33], in-plane CTE and out-of-plane CTE are the functions of $C_{V, a}$ and $C_{V, c}$ :

$$
\alpha_{a}=\frac{\gamma_{a}}{V}\left(S_{11}+S_{12}\right) \frac{2}{3} C_{V, a}+\frac{\gamma_{c}}{V} S_{13} \frac{1}{3} C_{V, c}+C T
$$

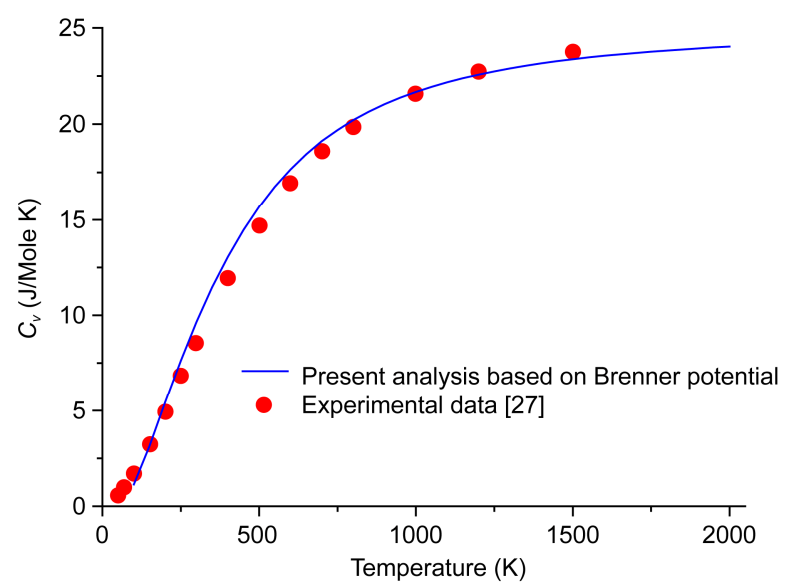

Figure 9 (Color online) Temperature dependence of specific heat $C_{v}$ for graphite predicted by eq. (49). The experimental data of graphite [27] are also shown. 


$$
\alpha_{c}=\frac{\gamma_{a}}{V}\left(S_{31}+S_{32}\right) \frac{2}{3} C_{V, a}+\frac{\gamma_{c}}{V} S_{33} \frac{1}{3} C_{V, c}+N T
$$

where $S_{11}, S_{12}, S_{13}$ and $S_{33}$ are the elastic flexibility coefficients of the crystal; $C$ and $N$ are the parameters; $V$ is the mole volume and equals $5.31 \mathrm{~cm}^{3} /$ mole; We set $\gamma_{a}=$ 2.05 and $r_{c}=1.33$ as the in-plane and out-of-plane Grüneisen parameters, respectively.

According to refs. [33,34], at room temperature, we have

$$
\begin{aligned}
& S_{11}+S_{12}=1.05 \times 10^{-13} \mathrm{~cm}^{2} / \mathrm{dyn}, \\
& S_{13}=-2.49 \times 10^{-13} \mathrm{~cm}^{2} / \mathrm{dyn}, \\
& S_{33}=26.3 \times 10^{-13} \mathrm{~cm}^{2} / \mathrm{dyn} .
\end{aligned}
$$

The present calculation results of in-plane and out-of-plane CTE are shown in Figures 10 and 11, respectively, which are in good agreement with experimental data $[27,35,36]$.

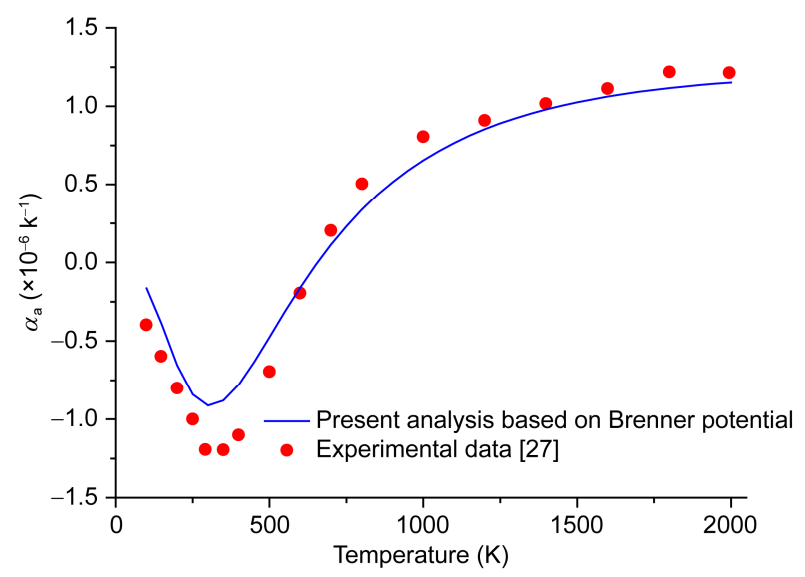

Figure 10 (Color online) Temperature dependence of in-plane CTE $\alpha_{\mathrm{a}}$ for graphite predicted by eq. (50). The experimental data of graphite [27] are also shown.

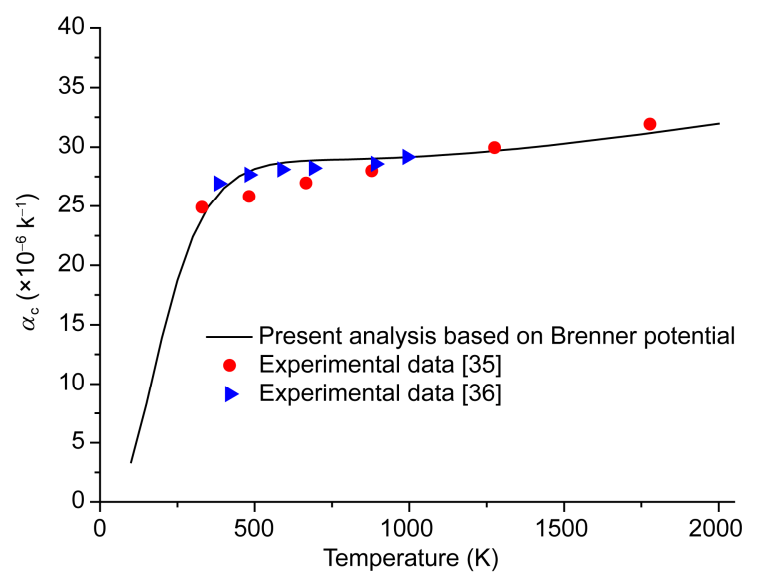

Figure 11 (Color online) Temperature dependence of in-plane CTE $\alpha_{\mathrm{c}}$ for graphite predicted by eq. (44) based on Brenner potential. The experimental data of graphite $[35,36]$ are also shown.

\section{3 lattice spacing of graphite}

Since the CTE of graphite in a-direction and c-direction are different, the a-spacing and c-spacing of graphite are also different, which can be expressed as the functions of $\alpha_{a}$ and $\alpha_{c}$, respectively:

$$
\begin{aligned}
& a(T)=a_{0}\left(1+\int_{0}^{T} \alpha_{a} \mathrm{~d} t\right), \\
& c(T)=c_{0}\left(1+\int_{0}^{T} \alpha_{c} \mathrm{~d} t\right),
\end{aligned}
$$

where $a_{0}=0.24618 \mathrm{~nm}, c_{0}=0.66818 \mathrm{~nm}$ [33]. They are lattice a-spacing and lattice c-spacing at absolute zero temperature respectively.

\subsection{The constitutive relation of graphite}

From eq. (46), one can obtain the thermal strain in a-direction $\varepsilon_{a}(T)$ and the thermal strain in c-direction $\varepsilon_{c}(T)$ as follow:

$$
\begin{aligned}
& \varepsilon_{a}(T)=\frac{a-a_{0}}{a_{0}}=\int_{0}^{T} \alpha_{a} \mathrm{~d} T, \\
& \varepsilon_{c}(T)=\frac{c-c_{0}}{c_{0}}=\int_{0}^{T} \alpha_{c} \mathrm{~d} T .
\end{aligned}
$$

Then the thermal strain tensor $\boldsymbol{E}^{*}$ for graphite can be expressed as:

$$
\boldsymbol{E}^{*}(T)=\left[\begin{array}{ccc}
\varepsilon_{a}(T) & 0 & 0 \\
0 & \varepsilon_{a}(T) & 0 \\
0 & 0 & \varepsilon_{c}(T)
\end{array}\right]
$$

Now the Cauchy stress for graphite can be expressed as follows:

$$
\begin{aligned}
& \boldsymbol{\sigma}=\frac{1}{V} \boldsymbol{F}^{e}\left\{\left[\frac{\partial U\left(\boldsymbol{E}^{e}\right)}{\partial \boldsymbol{E}^{e}}\right]\right\} \boldsymbol{F}^{e \mathrm{~T}}, \\
& \boldsymbol{E}^{e}=\left(\boldsymbol{U}^{* \mathrm{~T}}\right)^{-1}\left[\boldsymbol{E}-\boldsymbol{E}^{*}\right]\left(\boldsymbol{U}^{*}\right)^{-1} .
\end{aligned}
$$

The above equation is identical to eq. (16), but the thermal strain tensor $\boldsymbol{E}^{*}$ in eq. (49) is different from the thermal strain tensor $\boldsymbol{E}^{*}$ in eq. (26). The thermal strain tensor for copper is isotropic, while the thermal strain tensor for graphite is anisotropic.

Let us compare the present constitutive eq. (49) with the conventional constitutive equation. Using the Helmholtz free energy, the Cauchy stress takes the form [19-22]:

$$
\boldsymbol{\sigma}=\boldsymbol{F}\left\{\frac{\partial U_{t o t}}{\partial \boldsymbol{E}}+\sum_{i} \frac{\overline{E_{i}}}{\omega_{i}} \cdot \frac{\partial \omega_{i}}{\partial \boldsymbol{E}}\right\} \boldsymbol{F}^{\mathrm{T}} / V,
$$


where $\boldsymbol{F}$ is the deformation gradient.

From eq. (49), we can see that the present constitutive equation is quite compact and efficient in comparison with eq. (50). In eq. (49), the thermal strain $\boldsymbol{E}^{*}$ only depends on temperature $T$. Once we obtain the thermal strain $\boldsymbol{E}^{*}$, the stress tensor $\sigma$ can be directly derived from eq. (49). In eq. (50), all of the frequencies $\omega_{i}$ and its derivatives $\frac{\partial \omega_{i}}{\partial \boldsymbol{E}}$ are dependent to the applied strain $\boldsymbol{E}$. When the applied strain $\boldsymbol{E}$ increases or decreases, we should calculate all the frequencies and their derivatives again. Meanwhile, in order to guarantee the accuracy of the derivative of frequency $\frac{\partial \omega_{i}}{\partial \boldsymbol{E}}$, one should have a very accurate interatomic potential, which is a difficult task in practical application.

\section{Conclusion}

A continuum thermal stress theory for crystals at finite temperature is presented in this paper based on interatomic potential. The constitutive relation for cubic crystal copper and anisotropic crystal graphite is established via harmonic model and periodical boundary condition. The key idea is to introduce the thermal strain $\boldsymbol{E}^{*}$, which is independent of the applied strain $\boldsymbol{E}$. Free thermal expansion is an intrinsic property of crystals, when the temperature $T$ is rises. During free thermal expansion, the crystal is kept in case of stress free. Hence if the volume element of the crystal is stress free at the initial configuration, after free thermal expansion, the stress tensor $\sigma$ of the volume element of the crystal should be zero.

This work was supported by the National Natural Science Foundation of China (Grant Nos. 11021262, 11172303, 11132011) and National Basic Research Program of China (Grant No. 2012CB937500). The first author wishes to thank Mr. SHANG B.S. for his valuable advance.

1 Xu M W, Wang F, Zhao M S, et al. Molten hydroxides synthesis of hierarchical cobalt oxide nanostructure and its application as anode material for lithium ion batteries. Electrochim Acta, 2011, 56: 4876-4881

2 Sun $\mathrm{Y} \mathrm{M}$, Hu X L, Luo W, et al. Self-assembled hierarchical $\mathrm{MoO} 2 /$ graphene nanoarchitectures and their application as a high-performance anode material for lithium-ion batteries. Acs Nano, 2011, 5: 7100-7107

3 Pescini L, Lorenz H, Blick R H. Mechanical gating of coupled nanoelectromechanical resonators operating at radio frequency. Appl Phys Lett, 2003, 82: 352-354

4 Pantelic R S, Meyer J C, Kaiser U, et al. The application of graphene as a sample support in transmission electron microscopy. Solid State Commun, 2012, 152: 1375-1382

5 Shilkrot L E, Curtin W A, Miller R E. A coupled atomistic/continuum model of defects in solids. J Mech Phys Solids, 2002, 50: 20852106

6 Wagner G J, Liu W K. Coupling of atomistic and continuum simula- tions using a bridging scale decomposition. J Comput Phys, 2003, 190: $249-274$

7 Xiao S P, Belytschko T. A bridging domain method for coupling continua with molecular dynamics. Comput Method Appl Mech Eng, 2004, 193: 1645-1669

8 To A C, Li S F. Perfectly matched multiscale simulations. Phys Rev B, 2005, 72: 035414

9 Oden J T, Prudhomme S, Romkes A, et al. Multiscale modeling of physical phenomena: Adaptive control of models. J Sci Comput, 2006, 28: 2359-2389

10 Arroyo M, Belytschko T. An atomistic-based finite deformation membrane for single layer crystalline films. J Mech Phys Solids, 2002, 50: 1941-1977

11 Zhang P, Huang Y G, Geubelle P H, et al. On the continuum modeling of carbon nanotubes. Acta Mech Sin, 2002, 18: 528-536

12 Tadmor E B, Ortiz M, Phillips R. Quasicontinuum analysis of defects in solids. Philosophical Mag A, 1996, 73: 1529-1563

13 Tadmor E B, Smith G S, Bernstein N, et al. Mixed finite element and atomistic formulation for complex crystals. Phys Rev B, 1999, 59: 235-245

14 Shenoy V B, Miller R, Tadmor E B, et al. An adaptive finite element approach to atomic-scale mechanics - the quasicontinuum method. J Mech Phys Solids, 1999, 47: 611-642

15 Shenoy V, Phillips R. Finite temperature quasicontinuum methods. Multiscale Modelling Mater, 1999, 538: 465-471

16 Miller R E, Tadmor E B. The quasicontinuum method: Overview, applications and current directions. J Comput-aided Mater Des, 2002, 9: 203-239

17 Dupuy L M, Tadmor E B, Miller R E, et al. Fi-nite-temperature quasicontinuum: Molecular dynamics without all the atoms. Phys Rev Lett, 2005, 95: 060202

18 Tang Z, Aluru N R. Multiscale mechanical analysis of silicon nanostructures by combined finite temperature models. Comput Method Appl Mech Eng, 2008, 197: 3215-3234

19 Jiang H, Huang Y, Hwang K C. A finite-temperature continuum theory based on interatomic potentials. J Eng Mater-t Asme, 2005, 127: $408-416$

20 Tang Z, Zhao H, Li G, et al. Finite-temperature quasicontinuum method for multiscale analysis of silicon nanostructures. Phys Rev B, 2006, 74: 064110

21 Zhao H, Tang Z, Li G, et al. Quasiharmonic models for the calculation of thermodynamic properties of crystalline silicon under strain. $\mathrm{J}$ Appl Phys, 2006, 99: 064314

22 Tang Q H, Wang T C. Lattice wave theory of molecular dynamics. Sci China-Phys Mech Astron, 2011, 41: 214-220

23 Tang Q H, Wang T C, Shang B S, et al. Thermodynamic properties and constitutive relations of crystals at finite temperature. Sci China-Phys Mech Astron, 2012, 55: 918-926

24 Born M, Karman V T. On fluctuations in spatial grids. Physikalische Zeitschrift, 1912, 13: 297-309

25 Mishin Y, Mehl M, Papaconstantopoulos D, et al. Structural stability and lattice defects in copper: Ab initio, tight-binding, and embedded-atom calculations. Phys Rev B, 2001, 63: 224106

26 Ashcroft N W, Mermin N. Solid State Physics. Tokyo: Holt-Saunders, 1981

27 Billings B H, Gray D E. American Institute of Physics Handbook. Michigan: McGraw-Hill, 1972

28 Kittel C. Introduction to Solid State Physics. New York: John Wiley \& Sons, 1976

29 Nix F C, MacNair D. The thermal expansion of pure metals copper, gold, aluminum, nickel, and iron. Phys Rev, 1941, 60: 597-605

30 Overton W, Gaffney J. Temperature variation of the elastic constants of cubic elements. I. Copper. Phys Rev, 1955, 98: 969-977 
31 Brenner D W. Empirical potential for hydrocarbons for use in simulating the chemical vapor deposition of diamond films. Phys Rev B, 1990, 42: 9458-9471

32 Liu F, Tang Q H, Shang B S, et al. Simple optimized Brenner potential for thermodynamic properties of diamond. Philo Mag, 2012, 92: 500-515

33 Morgan W C. Thermal-expansion coefficients of graphite crystals. Carbon, 1972, 10: 73-79
34 Blakslee O L. Elastic constants of compression-annealed pyrolytic graphite. J Appl Phys, 1970, 41: 3373-3382

35 Steward E G, Cook B P, Kellett E A. The temperature dependence of the interlayer spacing in carbons of differing graphitic perfection. Acta Crystallographica, 1960, 13: 1101-1102

36 Nelson J B, Riley D P. The thermal expansion of graphite from 15-degrees-C to 800-degrees-C. Exp Proc Phys Soc London, 1945, 57: $477-486$ 